\title{
Evaluation of the Efficacy and Safety of Intravenous \\ Remdesivir in Adult Patients with Severe Pneumonia caused by COVID-19 virus Infection: study protocol for a Phase 3 Randomized, Double- blind, Placebo-controlled, Multicentre trial
}

Peter W Horby ( $\nabla$ peter.horby@ndm.ox.ac.uk )

University of Oxford https://orcid.org/0000-0002-9822-1586

Bin Cao

China-Japan Friendship Hospital

Yeming Wang

China-Japan Friendship Hospital

Chen Wang

Chinese Academy of Medical Sciences and Peking Union Medical College

Study protocol

Keywords: 2019-nCoV; clinical trial; remdesivir; antiviral; China

Posted Date: April 24th, 2020

DOI: https://doi.org/10.21203/rs.2.24058/v2

License: (9) This work is licensed under a Creative Commons Attribution 4.0 International License.

Read Full License

Version of Record: A version of this preprint was published at Trials on May 24th, 2020. See the published version at https://doi.org/10.1186/s13063-020-04352-9. 


\section{Abstract}

Background: A novel coronavirus emerged in Wuhan, Hubei Province, China towards the end of 2019 (SARS-CoV-2 or COVID-19 virus). Large scale spread within China and internationally led the World Health Organisation to declare a Public Health Emergency of International Concern on $30^{\text {th }}$ January 2020 . The clinical manifestations of COVID-19 virus infection include asymptomatic infection, mild upper respiratory symptoms, severe viral pneumonia with respiratory failure and even death. There are no antivirals of proven clinical efficacy in coronavirus infections. Remdesivir (GS-5734), a nucleoside analogue, has inhibitory effects on animal and human highly pathogenic coronaviruses, including MERSCoV and SARS-CoV, in in-vitro and in-vivo experiments. It is also inhibitory against the COVID-19 virus invitro. The aim of this study is to assess the efficacy and safety of remdesivir in adult patients with severe pneumonia caused by COVID-19 virus infection.

Methods: The protocol is prepared in accordance with the SPIRIT (Standard Protocol Items: Recommendations for Interventional Trials) guidelines. This is a phase 3, randomized, double-blind, placebo-controlled, multicentre trial. Adults ( $\geq 18$ years) with laboratory confirmed COVID-19 virus infection, and severe pneumonia signs or symptoms, and radiologically confirmed severe pneumonia are randomly assigned in a 2:1 ratio to intravenous remdesivir or placebo for 10 days. The primary endpoint is time to clinical improvement (censored at Day 28), defined as the time (in days) from randomization of study treatment (remdesivir or placebo) until a decline of two categories on a six-category ordinal scale of clinical status ( $1 \otimes$ discharged; $6 \otimes$ death) or live discharge from hospital. One interim analysis for efficacy and futility will be conducted once half of the total number of events required had been observed.

Discussion: This is the first randomized, placebo-controlled trial in 2019-nCoV. Enrolment began in sites in Wuhan, Hubei Province, China on $6^{\text {th }}$ February 2020.

Trial registration: ClinicalTrials.gov, NCT04257656, $6^{\text {th }}$ February 2020.

\section{Administrative Information}

Note: the numbers in curly brackets in this protocol refer to SPIRIT checklist item numbers. The order of the items has been modified to group similar items (see http://www.equator-network.org/reporting-guidelines/spirit-2013-statementdefining-standard-protocol-items-for-clinical-trials/). 


\begin{tabular}{|c|c|}
\hline Title [1] & $\begin{array}{l}\text { A Phase } 3 \text { Randomized, Double-blind, Placebo-controlled, Multicentre Study to Evaluate } \\
\text { the Efficacy and Safety of Intravenous Remdesivir in Adult Patients With Severe } \\
\text { Pneumonia caused by COVID-19 virus Infection }\end{array}$ \\
\hline $\begin{array}{l}\text { Trial registration } \\
\text { [2a and } 2 \mathrm{~b}]\end{array}$ & $\begin{array}{l}\text { Clinicaltrials.gov } \\
\text { NCT04257656 }\end{array}$ \\
\hline Protocol version [3] & $\begin{array}{l}\text { Version } 3.0 \\
09 \text { February } 2020\end{array}$ \\
\hline Funding [4] & $\begin{array}{l}\text { Funded by: } \\
\text { - Emergency project for novel coronavirus pneumonia of the Ministry of Science and } \\
\text { Technology (2020YFC0841300) } \\
\text { - Chinese Academy of Medical Sciences (CAMS) Innovation Fund for Medical Sciences } \\
\text { (CIFMS 2018-I2M-1-003) } \\
\text { - National Science Grant for Distinguished Young Scholars (81425001/H0104) } \\
\text { - The National Key Research and Development Program of China (2018YFC1200102), } \\
\text { - National Mega-projects for Infectious Diseases in China (2017ZX10103004 and } \\
\text { 2018ZX10305409). } \\
\text { Other support: } \\
\text { - International Severe Acute Respiratory and emerging Infections Consortium (ISARIC) } \\
\text { NIHR Senior Research Fellowship (TJ - 2015-08-001) }\end{array}$ \\
\hline Author details [5a] & $\begin{array}{l}\text { Department of Pulmonary and Critical Care Medicine, Center of Respiratory Medicine, } \\
\text { National Clinical Research Center for Respiratory Diseases, China-Japan Friendship } \\
\text { Hospital, Beijing, China } \\
\text { Jin Yin-tan Hospital; Wuhan, Hubei Province, China } \\
\text { Tongji Hospital, Tongji Medical College of Huazhong University of Science \& Technology, } \\
\text { Wuhan, Hubei Province, China } \\
\text { Wuhan Lung Hospital, Wuhan, Hubei Province, China } \\
\text { The Central Hospital of Wuhan, Wuhan, Hubei Province, China } \\
\text { Zhongnan Hospital of Wuhan University, Wuhan, Hubei Province, China } \\
\text { Renmin Hospital of Wuhan University, Wuhan, Hubei Province, China } \\
\text { Union Hospital, Tongji Medical College of Huazhong University of Science \& Technology, } \\
\text { Wuhan, Hubei Province, China } \\
\text { Wuhan First hospital, Wuhan, Hubei Province, China }\end{array}$ \\
\hline
\end{tabular}




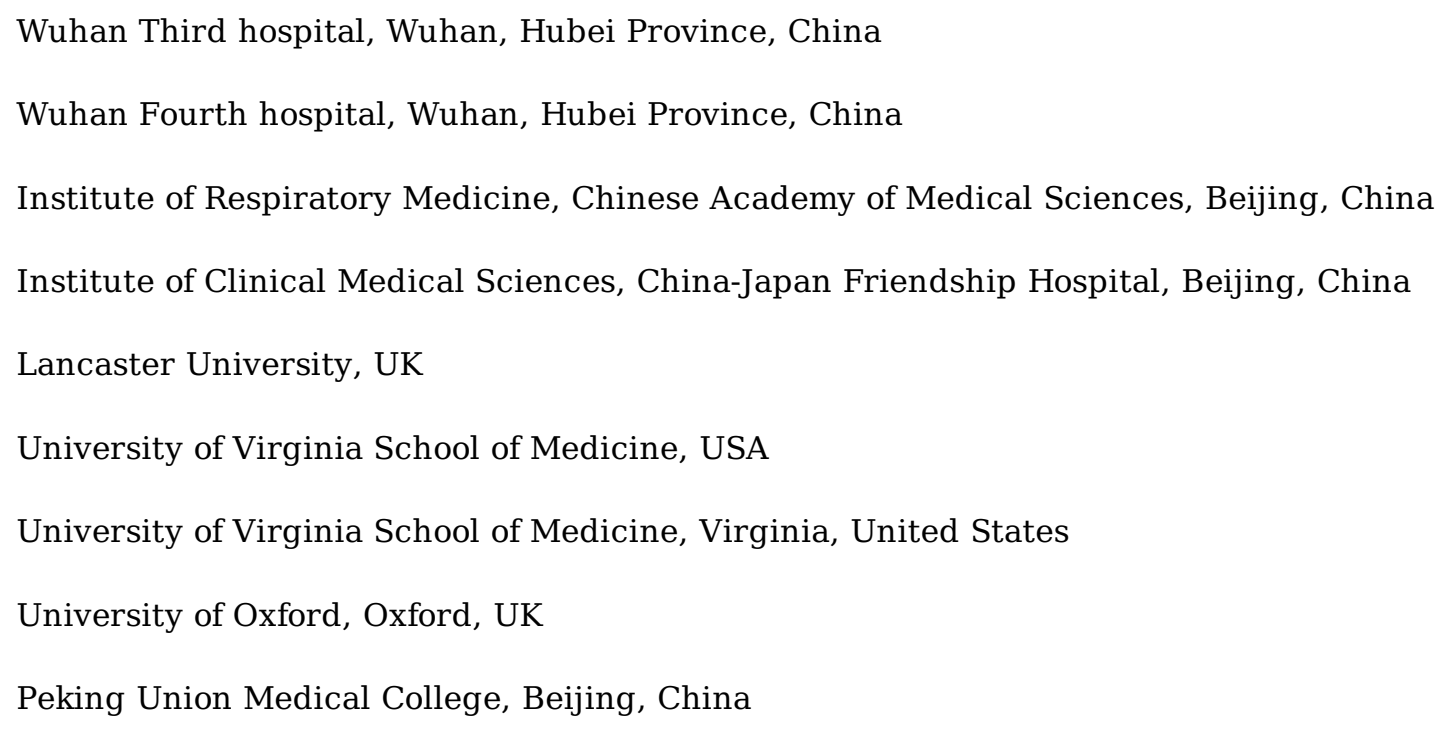

The study sponsor and funders had no role in study design; collection, management, analysis, and interpretation of data; writing of the report; and the decision to submit the report for publication.

\section{Introduction}

\section{Background and rationale [6a]}

In December 2019, Wuhan City, Hubei Province experienced an outbreak of pneumonia of unknown cause. The outbreak may have started with zoonotic transmission of the virus in a large seafood market involving live wildlife trading, with subsequent sustained transmission from person to person. On $7^{\text {th }}$ January 2020 a previously unidentified betacoronavirus, later named SARS-CoV-2 virus (or COVID-19 virus), was identified by the Chinese Center for Disease Control and Prevention (China CDC) as the aetiological agent. ${ }^{1}$ The COVID-19 virus is probably derived originally from bats and amongst coronaviruses know to infect humans, is most closely related, but distinct from, the SARS coronavirus. The clinical manifestations of COVID-19 virus infection include asymptomatic infection, mild upper respiratory symptoms, severe viral pneumonia with respiratory failure and even death. Although the risk of severe illness is not yet clear, hospitals in areas with significant community transmission have experienced a major increase in the number of hospitalized pneumonia patients, with the frequency of severe disease in hospitalised patients being as a high as $30 \% .{ }^{2-4}$ The progression from prodromes 
(usually fever, fatigue and cough) to severe pneumonia requiring oxygen support, mechanical ventilation, or ECMO is most commonly seen in the second week following onset of symptoms of a viral infection. ${ }^{2}$ The kinetics of viral replication in the respiratory tract have not been well characterized, but this relatively slow progression provides a potential time window and opportunity for antiviral therapies to influence the course of disease.

Remdesivir (GS-5734) is a monophosphoramidate prodrug of an adenosine analogue (GS-441524), and has broad action against a range of RNA viruses. ${ }^{5}$ It has been identified as the most promising therapeutic agent for evaluation in the treatment of COVID19 by an expert committee convened by the WHO R\&D Blueprint. ${ }^{6}$ The primary mechanism of action is the intracellular incorporation of the pharmacologically active nucleoside triphosphate form into nascent RNA chains by the viral RNAdependent RNA-polymerase, causing premature RNA chain termination. ${ }^{7-9}$ In-vitro experiments have shown that remdesivir inhibits bat coronaviruses, endemic human coronavirus (OC43, 229E), and the human pathogenic coronaviruses, MERS-CoV, SARS-CoV, and COVID-19 virus. ${ }^{10-13}$ Remdesivir has shown preventive and therapeutic effects in a mouse model of SARS-CoV. ${ }^{10}$ In a MERS-CoV mouse model, prophylactic and therapeutic administration of remdesivir improved lung function, decreased lung viral load and reduced severe lung pathological findings. ${ }^{14}$ Remdesivir has also shown prophylactic efficacy in MERS-CoV-infected Indian rhesus monkeys (personal communication: Gilead Sciences Inc.).

Evaluation of intravenous remdesivir tolerance and safety in 94 healthy adult volunteers has found it to be generally well tolerated and have an acceptable safety profile. The only significant adverse effect were transient grade 1 or grade 2 increases in AST and ALT (personal communication: Gilead Sciences Inc.). Further clinical experience was obtained through a randomized controlled trial in patients with Ebola virus disease. In this trial 175 patients received intravenous remdesivir with a loading dose on day 1 (200 $\mathrm{mg}$ in adults, and adjusted for body weight in pediatric patients), followed by a daily maintenance dose (100 mg in adults) starting on day 2 and continuing for 9 to 13 days. The only reported serious adverse events (SAE) was one patient who experienced hypotension that resulted in cessation of the remdesivir loading dose, followed rapidly by cardiac arrest. However, the cause of death could not be distinguished from the patient's underlying severe Ebola virus disease. ${ }^{15}$

Given the in-vitro and in-vivo effectiveness of remdesivir against a range of pathogenic human coronaviruses and the acceptable tolerance and safety in humans, we developed this trial with the objective of evaluating the safety and efficacy of intravenous remdesivir in adults with severe pneumonia caused by COVID-19 virus infection.

\section{Objectives [7]}

To evaluate the efficacy and safety of intravenous remdesivir combined with standard of care compared with standard care alone in adult patients with severe pneumonia caused by COVID-19 virus infection. 


\section{Trial design [8]}

This is a phase 3, parallel group, randomized, double-blind, placebo-controlled, superiority, multicentre trial. The allocation ratio is $2: 1$ in favour of remdesivir to maximise learning about the experimental treatment while allowing a wider pool of patients access to the experimental treatment in order to support recruitment.

\section{Methods}

\section{Participants, interventions and outcomes}

\section{Study setting [9]}

Hospitals in Wuhan city, Hubei Province, People's Republic of China. Study sites included Jin Yin-tan Hospital; Wuhan, Hubei Province, China; Tongji Hospital, Tongji Medical College of Huazhong University of Science \& Technology; Wuhan Lung Hospital; The Central Hospital of Wuhan; Zhongnan Hospital of Wuhan University; Renmin Hospital of Wuhan University; Union Hospital, Tongji Medical College of Huazhong University of Science \& Technology; Wuhan First hospital; Wuhan Third hospital; Wuhan Fourth hospital.

\section{Eligibility criteria [10]}

Inclusion Criteria:

1. Age $\geq 18$ years at time of signing Informed Consent Form.

2. Laboratory (RT-PCR) confirmed infection with COVID-19 virus.

3. Pneumonia confirmed with chest imaging.

4. Hospitalized with a SPO2 $\leq 94 \%$ on room air or Pa02/FiO2 $\leq 300 \mathrm{mmHg}$.

5 . $\leq 12$ days since symptoms onset.

6. Willingness of study participant to accept randomization to any assigned treatment arm.

7. Males, and females of child-bearing age must agree to use effective birth control measures (hormone method, barrier method, or abstinence) during the trial and at least 7 days after the last medication.

8. Must agree not to enrol in any other study of an antiviral agent prior to completing the 28-day followup.

\section{Exclusion Criteria:}

1. Physician makes a decision that trial involvement is not in patients' best interest, or any condition that does not allow the protocol to be followed safely.

2. Known severe liver disease (e.g. cirrhosis, $A L T>5 \times U L N$, or $A S T>5 \times U L N)$. 
3. Pregnant or breastfeeding, or positive pregnancy test in women of childbearing age.

4. Patients with known severe renal impairment (estimated glomerular filtration rate $\leq 30 \mathrm{ml} / \mathrm{min} / 1.73$ $\mathrm{m}^{2}$ ); or patients receiving continuous renal replacement therapy, hemodialysis, or peritoneal dialysis.

5 . Will be transferred to any other hospital which is not a study site within 72 hours.

6. Receipt of any experimental treatment for COVID-19 virus infection within 30 days prior to screening.

\section{Who will take informed consent? [26a]}

Informed consent will be obtained from eligible patients or their substitute decision-makers (for patients lacking decision-making capacity) by study physicians or other trial staff with delegated responsibility.

\section{Additional consent provisions for collection and use of participant data and biological specimens [26b]}

The consent form includes provisions for research data and samples, and residual clinical blood samples to be stored for future scientific research on COVID-19 virus disease.

\section{Interventions}

\section{Explanation for the choice of comparators [6b]}

The active arm is intravenous remdesivir in addition to routine supportive care. Remdesivir was chosen for evaluation because of in vitro activity against SARS-CoV, MERS-CoV, and SARS-CoV-2, and in vitro activity against SARS-CoV and MERS-CoV. There are safety data for remdesivir in healthy human volunteers and individuals with Ebola virus disease. These data suggest that remdesivir is generally well tolerated and has an acceptable safety profile. It has been identified as the most promising therapeutic agent for treatment of COVID19 by the WHO R\&D Blueprint. ${ }^{6}$ The dose of remdesivir was selected on the recommendation of Gilead Sciences Inc. based on known pharmacokinetics and dose ranging studies in healthy volunteers. The control arm is placebo in addition to routine supportive care. There are no proven antiviral therapies for SARS-CoV-2 to include as an active control comparator.

\section{Intervention description [11a]}

- Group A (experimental group):

Loading dose: Remdesivir $200 \mathrm{mg}$ in $350 \mathrm{ml}$ normal saline ( $0.9 \%$ sodium chloride) single daily dose infused intravenously over approximately 30 to 60 minutes (with a target time of 30 minutes) for 1 day.

Maintenance dose: Remdesivir $100 \mathrm{mg}$ in $250 \mathrm{ml}$ normal saline ( $0.9 \%$ sodium chloride) single daily dose infused intravenously over approximately 30 to 60 minutes (with a target time of 30 minutes) for 9 days.

- Group B (control group): 
Loading dose: Placebo in $350 \mathrm{ml}$ normal saline ( $0.9 \%$ sodium chloride) single daily dose infused intravenously over approximately 30 to 60 minutes (with a target time of 30 minutes) for 1 day.

Maintenance dose: Placebo in $250 \mathrm{ml}$ normal saline ( $0.9 \%$ sodium chloride) single daily dose infused intravenously over approximately 30 to 60 minutes (with a target time of 30 minutes) for 9 days.

\section{Criteria for discontinuing or modifying allocated interventions [11b]}

1. Any serious or intolerable adverse event which, in the investigators' judgement, requires withdrawal of the subject from the study. This may include: Drug intolerance or an unacceptable adverse event $(\mathrm{AE})$; any severe grade $4 \mathrm{AE}$, regardless of whether the $\mathrm{AE}$ is considered likely related to the study drug or not; significant results of laboratory tests which may require withdrawal of the participant, at the discretion of the researchers and the clinician; allergic reactions (including oropharyngeal oedema, severe rash, bronchospasm, and immediate-type allergic reactions); acute hepatitis[1]; stage III or above acute kidney injury.

2. Investigator request for the subject to be withdrawn from the study.

\section{Strategies to improve adherence to interventions [11c]}

Not applicable, since active agent and placebo are administrated intravenously by health care professionals.

\section{Relevant concomitant care permitted or prohibited during the trial [11d]}

This study seeks to evaluate the effect of remdesivir in addition to standard of care alone, rather than an effect that might be altered by other experimental treatments. Therefore, all concomitant care and interventions are permitted other than concomitant receipt of any other experimental treatment.

\section{Provisions for post-trial care [30]}

No special arrangements for post-trial care are anticipated.

\section{Outcomes [12]}

\section{Primary Outcome Measures:}

1. Time to Clinical Improvement (TTCI) [Censored at Day 28]

TTCl is defined as the time (in days) from randomization of study treatment (active or placebo) until a

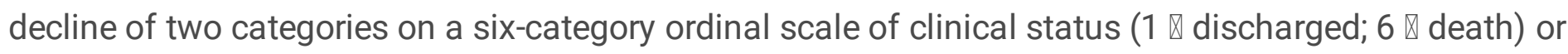
live hospital discharge, whichever comes first.

Six-category ordinal scale: 
$6 \rrbracket$ death; 5 \ hospitalization, requiring ECMO and/or IMV; 4 \hospitalization, requiring NIV and/or HFNC therapy; $3=$ hospitalization, requiring supplemental oxygen (but not NIV/HFNC); 2 = hospitalization, not requiring supplemental oxygen; 1 = hospital discharge

Abbreviation: ECMO, extracorporeal membrane oxygenation; IMV, invasive mechanical ventilation; NIV, non-invasive mechanical ventilation; HFNC, High-flow nasal cannula.

\section{Secondary Outcome Measures:}

1. Percentage of subjects in each clinical category on the six-category ordinal scale [Time Frame: days $7,14,21$, and 28].

2. Time to disease improvement, defined as the time to Hospital Discharge, OR NEWS2 (National Early Warning Score 2) of $\leq 2$ maintained for 24 hours [Time Frame: up to 28 days].

3. All cause mortality [Time Frame: up to 28 days].

4. Duration (days) of invasive mechanical ventilation [Time Frame: up to 28 days].

5. Duration (days) of supplemental oxygenation [Time Frame: up to 28 days].

6. Length of hospital stay (days) [Time Frame: up to 28 days].

7. Incidence of nosocomial secondary infection.

8. Time to COVID-19 virus RT-PCR negativity in upper and lower respiratory tract specimens [Time Frame: up to 28 days].

9. Change (reduction) in COVID-19 viral RNA load in upper and lower respiratory tract specimens as assessed by area under viral load curve [Time Frame: up to 28 days].

10. Pharmacokinetics parameters of remdesivir or its active metabolites.

11. Frequency of serious adverse events [Time Frame: up to 28 days].

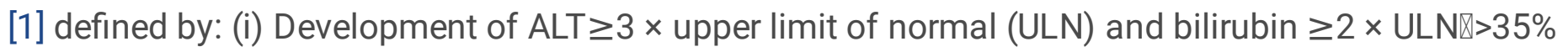
conjugated bilirubin. If conjugated/unconjugated bilirubin is not available, urobilinogen should be tested for on a test strip, and a positive result to be indicative of $>35 \%$ conjugated bilirubin. If neither tests are available, the study drug should be discontinued. (ii) $A L T \geq 5 \times U L N$. (iii) $A L T \geq 3 \times U L N$ and onset of symptoms of hepatitis, such as fatigue, nausea, vomiting, right upper quadrant pain, fever, rash or eosinophilia.

\section{Participant timeline [13]}




\begin{tabular}{|c|c|c|c|c|c|}
\hline \multirow[b]{2}{*}{ Process } & \multirow{2}{*}{$\begin{array}{c}\text { Screening/Baseline/Randomization } \\
\text { B/L/D0 }\end{array}$} & \multirow{2}{*}{$\begin{array}{c}\begin{array}{c}\text { Treatment } \\
\text { phase }\end{array} \\
\text { D1-D10 }\end{array}$} & \multicolumn{3}{|c|}{ Follow-up phase } \\
\hline & & & $\begin{array}{c}\text { D14 } \\
\pm 1\end{array}$ & $\begin{array}{c}\mathrm{D} 21 \\
\pm 3\end{array}$ & $\begin{array}{c}\mathrm{D} 28 \\
\pm 3\end{array}$ \\
\hline Inclusion/exclusion criteria & $\mathrm{X}$ & & & & \\
\hline Written informed consent & $\mathrm{X}$ & & & & \\
\hline Demographics & $\mathrm{X}$ & & & & \\
\hline $\begin{array}{l}\text { Assessment of concomitant chronic } \\
\text { diseases }\end{array}$ & $\mathrm{X}$ & & & & \\
\hline \multicolumn{6}{|l|}{ Efficacy / safety assessment } \\
\hline ECG & $\mathrm{X}$ & & $\mathrm{X}$ & & \\
\hline Chest imaging & $\mathrm{X}$ & $\begin{array}{l}\mathrm{X} \text { (only on } \\
\mathrm{D} 10)\end{array}$ & & & $\mathrm{X}$ \\
\hline $\begin{array}{l}\text { Vital signs } \\
\text { (Body temperature, heart rate, blood } \\
\text { pressure, breathing rate, oxygen } \\
\text { saturation, etc.) }\end{array}$ & $\mathrm{X}$ & $\mathrm{X}$ & $\mathrm{X}$ & $\mathrm{X}$ & $\mathrm{X}$ \\
\hline $\begin{array}{l}\text { Clinical symptoms assessment } \\
\text { (fever, cough, diarrhea, dyspnea) }\end{array}$ & $\mathrm{X}$ & $\mathrm{X}$ & $\mathrm{X}$ & $\mathrm{X}$ & $\mathrm{X}$ \\
\hline Primary endpoint assessment & $\mathrm{X}$ & $\mathrm{X}$ & $\mathrm{X}$ & $\mathrm{X}$ & $\mathrm{X}$ \\
\hline Adverse events & $\mathrm{X}$ & $\mathrm{X}$ & $\mathrm{X}$ & $\mathrm{X}$ & $\mathrm{X}$ \\
\hline \multicolumn{6}{|l|}{ Laboratory test } \\
\hline $\begin{array}{l}\text { Diagnosis } \\
\text { (clinical diagnosis, antibody diagnosis, or } \\
\text { pathogen diagnosis) }\end{array}$ & $\mathrm{X}$ & & & & \\
\hline $\begin{array}{l}\text { Whole blood count } \\
\text { (sampling time) }\end{array}$ & $\mathrm{X}$ & $\begin{array}{l}\mathrm{X}(\text { only on D3, } \\
\text { D7, and D10) }\end{array}$ & & & \\
\hline $\begin{array}{l}\text { Coagulation routine } \\
\text { (sampling time) }\end{array}$ & $\mathrm{X}$ & $\begin{array}{l}\text { X (only on D3, } \\
\text { D7, and D10) }\end{array}$ & & & \\
\hline $\begin{array}{l}\text { Kidney and liver function tests } \\
\text { (sampling time) }\end{array}$ & $\mathrm{X}$ & $\begin{array}{l}\text { X (only on D3, } \\
\text { D7, and D10) }\end{array}$ & & & \\
\hline Arterial blood gas analysis & $\mathrm{X}$ & & & & \\
\hline $\begin{array}{l}\text { Pregnancy test } \\
\text { (urine/blood, for women of childbearing } \\
\text { age only) }\end{array}$ & $\mathrm{X}$ & & & & \\
\hline Nasopharyngeal/ oropharyngeal swab & $\mathrm{X}$ & $\mathrm{X}$ & $\mathrm{X}$ & $\mathrm{X}$ & $\mathrm{X}$ \\
\hline $\begin{array}{l}\text { Lower respiratory tract specimen } \\
\text { (sputum / tracheal aspirate / bronchial } \\
\text { alveolar lavage fluid) if available }\end{array}$ & $\mathrm{X}$ & $\mathrm{X}$ & $\mathrm{X}$ & $\mathrm{X}$ & $\mathrm{X}$ \\
\hline Feces / anal swabs & $\mathrm{X}$ & $\mathrm{X}$ & $\mathrm{X}$ & $\mathrm{X}$ & $\mathrm{X}$ \\
\hline $\begin{array}{l}\text { Pharmacokinetics sampling } \\
\text { (approximately } 4 \text { ml whole blood) }\end{array}$ & & $\begin{array}{l}\text { X (only on D1, } \\
\text { D3, D7 (as } \\
\text { needed), and } \\
\text { D10) } \\
\end{array}$ & & & \\
\hline \multicolumn{6}{|l|}{ Interventions } \\
\hline Remdesivir / placebo & & $\mathrm{X}$ & & & \\
\hline $\begin{array}{l}\text { Concomitant medications } \\
\text { (record only) }\end{array}$ & $\mathrm{X}$ & $\mathrm{X}$ & $\mathrm{X}$ & $\mathrm{X}$ & $\mathrm{X}$ \\
\hline
\end{tabular}




\section{Sample size [14]}

The primary outcome is time to clinical improvement (TTCI) [censored at day 28]. An interim analysis for futility and efficacy is planned at the mid-point of the trial. At this point the sample size may also be reassessed. A one-sided type I error of $2.5 \%$ across the two stages of the trial and a power of $80 \%$ if the hazard ratio comparing remdesivir to placebo is 1.4 is used. This hazard ratio corresponds to a reduction in time to clinical improvement to 15 days on remdesivir if the time to clinical improvement on standard of care was 21 days. Triangular boundaries ${ }^{16}$ are used to account for the multiple looks at the data and allowance for the 2:1 randomization in favour of remdesivir is made. Under these assumptions, the total number of events required in this study is 325 .

Assuming an $80 \%$ event rate within 28 days across both arms and a drop-out rate of $10 \%$ implies that approximately 453 patients are to be recruited for this trial (151 on placebo and 302 on remdesivir).

\section{Recruitment [15]}

Patients will be recruited from the designated hospitals for COVID-19 virus patients.

\section{Assignment of interventions: allocation}

\section{Sequence generation [16a]}

A permuted block (30 patients per block) randomization sequence, including stratification, is prepared by a statistician not involved in the trial using SAS software, version 9.4 (SAS Institute). Patient randomisation is stratified based on respiratory support methods at the time of enrolment: (1) no oxygen support, oxygen support with nasal duct or mask; (2) high-flow oxygen, non-invasive ventilation, invasive ventilation/ECMO.

\section{Concealment mechanism [16b]}

Eligible patients were allocated to receive medication in individually numbered packs, according to the sequential order provided by the randomization centre (Jin Yin-Tan Hospital central pharmacy). Remdesivir and placebo are pre-blinded and stored in a secure area in the pharmacy at a temperature strictly controlled according to the protocol. An independent pharmacist is assigned to dispense the study drug in water-proof, sealed, opaque bags.

\section{Implementation [16c]}

Patients will receive medication in individually numbered packs, according to the sequential order provided by the randomization centre. Opaque envelopes were provided for emergency unbinding events. The allocation sequence was generated by the institutes of Materia Medica, CAMS \& PUMC. Participants 
are enrolled by the investigators of each study site. A pharmacist in the central pharmacy assigns participants to interventions.

\section{Assignment of interventions: Blinding}

\section{Who will be blinded [17a]}

This is a double-blind trial. Trial participants, investigators, care providers, outcome assessors, and data analysts are all blinded. Treatment allocation will only be unblinded after database lock.

\section{Procedure for unblinding if needed [17b]}

Unblinding is permissible when investigators believe that there is a very strong need to know the study drug allocation in order to perform any agent treatment/action. Whenever possible, unblinding will only be conducted after discussion with the study principal investigator. The procedure for revealing a participant's allocated intervention is as follows: (1) investigators confirm that the patient meets the criteria of unblinding according to the protocol; (2) the independent pharmacist opens the sealed envelope and informs the investigators of the allocation; (3) information about the date, time, and reason of unblinding will be recorded in the EDC and the envelope; (4) the envelope must be sealed again as soon as possible and securely stored along with the primary files of the subject.

\section{Data collection and management}

\section{Plans for assessment and collection of outcomes [18a]}

Investigators are responsible for assessment and collection of outcome, baseline, and other trial data, and the data are double checked by the clinical research coordinators and clinical research associates as well as data managers. Nasopharyngeal/oropharyngeal swabs, lower respiratory tract specimens (sputum / tracheal aspirate / bronchial alveolar lavage fluid), feces / anal swabs and pharmacokinetics samples (whole blood) will be sent to a central laboratory, where tests will performed according to laboratory SOPs. Data collection forms can be found in https://edc.clinflash.net/login.

For the efficacy outcome measures, two scales [six-category ordinal scale of clinical status, and NEWS2 (National Early Warning Score 2)] are used. Investigators will be trained to use these scales.

\section{Plans to promote participant retention and complete follow-up [18b]}

Subjects may voluntarily withdraw from the trial at any time. This decision must be communicated with and reviewed by investigators. Staff at study sites should explain to these subjects the importance of staying in the study for the full duration of follow-up of this trial. For any withdrawal from this trial, investigators must fill in the withdrawal reason in the eCRF and try to complete all remaining assessments up to Day 28 after randomization. For subjects who withdraw due to adverse events (AEs), investigators should closely follow up their AEs until these AEs disappear, return to the baseline state or 
the AE condition is stable. If subjects are lost to follow-up, existing data collected up until the time of loss to follow up will be used.

Investigators may request for subjects to be withdrawn from receiving the study drugs for clinical or other reasons. In this case, subjects should remain in the trial after terminating the trial treatment and be followed up for all scheduled visits and the corresponding data recorded.

\section{Data management [19]}

The EDC system will be used for data management, including data entry, query, coding, security, and storage. The data manager built the database according to the protocol, and at the same time made logical verification settings for data validity, so as to verify the data. The data of each enrolled subject (including discontinued subjects) will be recorded into database/eCRF rapidly, completely, and accurately. Each completed eCRF will be reviewed and signed (electronically) by the investigators. The data manager will check the data and ask investigators to resolve any queries identified. The data administrator will proofread and modify the data according to investigators' answers. The data are securely stored in the EDC system, with the server located in Zhejiang province, China.

\section{Confidentiality [27]}

Subjects' data collected in case report forms (CRFs) will be identified by a study subject ID only. Samples collected through the study will be identified by a study subject ID only. The log linking the study subject ID with the patient identifying information will be held at each recruitment site. In some emergent or rare event that, for safety or regulatory reasons, it may be necessary to identify a subject, the sponsor and investigators were bound to keep this information confidential.

\section{Plans for collection, laboratory evaluation and storage of biological specimens for genetic or molecular analysis in this trial/future use [33]}

Nasopharyngeal/oropharyngeal swabs, lower respiratory tract specimens (sputum / tracheal aspirate / bronchial alveolar lavage fluid), feces / anal swabs and pharmacokinetics samples (whole blood) will collected by investigators or designees, marked with the study subject ID, and then immediately send to the central laboratory for coronavirus nucleic acid detection or remdesivir/active metabolites concentration detection. Sample collection, handling, labelling, storage, shipping, processing, etc. will performed according to the requirements of the operation manual in the central laboratory.

Central laboratory: Teddy Clinical Research Laboratory (Shanghai) Limited.

\section{Statistical methods}

Statistical methods for primary and secondary outcomes [20a] 
The primary outcome is time to clinical improvement up to day 28 of patients on remdesivir compared to patients receiving placebo. Patients will be randomized in a ratio of 2:1 to receive either remdesivir or placebo. All reporting will adhere to the CONSORT guideline as well as the corresponding extension for adaptive clinical trials. ${ }^{17,18}$

The final analysis of the primary endpoint will use a Cox proportional hazards model following the intention to treat principle. The final analysis will be conducted once a total of 325 events have occurred across both treatment groups. Under the assumption of an $80 \%$ event rate within 28 days and accounting for $10 \%$ dropout, this means that the maximum number of patients to be recruited is approximately 453 patients. A standardized test statistic is then found on the basis of this model and if this test statistic exceeds 2.095 , then it will be concluded that remdesivir is significantly better than placebo. Median unbiased effect estimates and confidence intervals accounting for the interim analysis will be reported. ${ }^{19}$ Analysis of secondary endpoints will use a logistic model for binary variables such as mortality and a Cox proportional hazards model for time to event endpoints. A statistical analysis plan (SAP) providing all details of the analyses to be undertaken will be completed before any data analysis will be conducted.

\section{Interim analyses [21b]}

An interim analysis for efficacy and lack of benefit will be undertaken once a total of 163 events have been observed across all treatment group. For the interim analysis the primary endpoint will be analysed using a Cox proportional hazards model using the intention to treat principle. A standardized test statistic is then found on the basis of this model and if this test statistic falls below 0.741 , the trial will be stopped for lack of benefit of remdesivir. If the test statistic exceeds 2.222 , the trial will terminate with the conclusion, that remdesivir is significantly better than control. If the test statistics falls between 0.741 and 2.222, the trial continues to the second stage and a sample size re-estimation on the basis of the observed event rate will take place. The interim analysis is to be conducted by an independent statistician and reviewed by the DSMC. Should the trial stop for futility or efficacy the median unbiased estimate as well as confidence intervals accounting for the interim analysis will be reported. ${ }^{19}$

\section{Methods for additional analyses (e.g. subgroup analyses) [20b]}

Separate supporting analysis will be undertaken for gender and age groups ( $<60$ and over 60 years of age at randomisation). These analyses will use a Cox proportional hazards model of the primary endpoint. Additionally, mortality will be analysed for each of these subgroups using a logistic regression model.

\section{Methods in analysis to handle protocol non-adherence and any statistical methods to handle missing data [20c]}


Multiple imputation will be used for missing data. The primary analysis will use the intention to treat principle and a per protocol analysis will be undertaken to assess the robustness of the findings.

Plans to give access to the full protocol, participant level-data and statistical code [31c]

These plans are not yet in place.

\section{Oversight and monitoring}

Composition of the coordinating centre and trial steering committee [5d]

\section{Coordinating Centre}

The study is led by the China-Japan Friendship Hospital, Wuhan Jinyintan Hospital, Wuhan Central Hospital, Huazhong University of Science and Technology Tongji Hospital, Wuhan University Zhongnan Hospital, Wuhan Pulmonary Hospital, Wuhan University People's Hospital, Wuhan Union Hospital and other hospitals.

\section{Trial steering committee}

Chen Wang, President of Pekin Union Medical College

Bin Cao (China-Japan Friendship Hospital), deputy team leader of NCP Group

Dingyu zhang (Jinyintan Hospital), Director of NCP Designated Hospital.

Ke Wang, Director of Institute of Medicine, Chinese Academy of Medical Sciences.

\section{Trial Operation Committee}

Yeming Wang, Fei Zhou, China-Japan Friendship Hospital.

Ying Liu, Shunan Ruan, Wen Liu, Jinyintan Hospital.

\section{Trial Monitoring}

Hangzhou Tigermed Consulting Co., Ltd.

\section{Data management team}

Hangzhou Tigermed Consulting Co., Ltd

\section{Clinical Research Organisation}

Hangzhou Tigermed Consulting Co., Ltd 


\section{Composition of the data monitoring committee, its role and reporting structure [21a]}

The Independent Data Safety Monitoring Board (DSMB) in this study is responsible for reviewing the reports regarding the safety of the study patients and protocol adherence and making recommendations to continue or terminate the study or modify sample size on the basis of the results from the interim analysis. The DSMB members are all independent from the Sponsor and have no financial or other conflicts of interest.

\section{DMC Members}

\begin{tabular}{|l|l|l|}
\hline Name & Role & Position \\
\hline Jieming Qu & Chair & $\begin{array}{l}\text { Professor of Respiratory Medicine \& President, Ruijin Hospital Shanghai Jiao Tong } \\
\text { University School of Medicine }\end{array}$ \\
\hline $\begin{array}{l}\text { Weichung } \\
\text { Joe Shih }\end{array}$ & member & Director of Biometrics Division of the Cancer Institute of New Jersey, Rutgers University \\
\hline $\begin{array}{l}\text { Rob } \\
\text { Fowler }\end{array}$ & member & $\begin{array}{l}\text { Senior scientist, Evaluative Clinical Sciences, Trauma, Emergency \& Critical Care Research } \\
\text { Program, Sunnybrook Research Institute, Toronto, Canada }\end{array}$ \\
\hline $\begin{array}{l}\text { Rory } \\
\text { Collins }\end{array}$ & member & Head, Nuffield Department of Population Health, University of Oxford, UK \\
\hline Chen Yao & member & Biometrics Division of Peking University \\
\hline
\end{tabular}

\section{Adverse event reporting and harms [22]}

AEs and SAEs will be collected from the time of informed consent to Day 28. SAEs occurring after Day 28 \pm 3 will be reported if investigators determine that these SAEs are related to the study drugs. SAEs will be followed-up until the SAE has subsided, returned to baseline, or is stable.

Investigators or designees are responsible for collecting, assessing, reporting, and managing AEs. AEs will be fully investigated and recorded in detail in the CRF, including onset date, the duration of $A E$, symptoms/signs, severity, action taken to manage the $A E$, relationship with the study drug, outcome of the $A E$, and date of outcome assessment (if outcome was other than recovering, not recovered, or unknown).

\section{Frequency and plans for auditing trial conduct [23]}

The contract research organization (Hangzhou Tigermed Consulting Co., Ltd.

During the trial, the principal investigator will inform the Independent Ethics Committee (IEC) of any revision or modification of the protocol. The revision or modification will only be implemented after receiving IEC's approval, unless it is necessary to be implemented in order to eliminate immediate and direct harm to patients, in which case, the IEC will be informed as soon as possible. 
After any protocol amendment, the informed consent form and any other written information provided to subjects will be updated as necessary. Investigators will inform subjects in time and ask them to sign the revised informed consent form to confirm their participation. The updated informed consent form must be approved by the IEC before implementation.

\section{Dissemination plans [31a]}

We will communicate trial results to national and international health authorities, healthcare professionals, the public, and other relevant groups as soon as the trial results are available.

\section{Discussion}

This study is the first double blind, placebo-controlled trial of an experimental therapeutic for COVID-19 virus. Whilst the COVID-19 virus epidemic has been declared a public health emergency of international concern, this does not negate the need to generate robust evidence in which healthcare professionals, patients, their families, and public health authorities can have confidence. In past epidemics, unproven treatments have been used on a compassionate use basis, in observational studies, or in underpowered trials. The result is uncertainty and ongoing treatment dilemmas. Randomised, placebo-controlled trials are well established as the best way to minimise bias and confounding and produce reliable evidence. Such trials can be implemented even in the most difficult circumstances; in fact, the difficult circumstances themselves make it even more important that patient care is not driven by fear and rumour but by science and evidence. During health emergencies doctors and politicians are under enormous pressure to find cures to save lives and stop the epidemic, whilst patients are willing to try anything in the face of a frightening and unknown threat. We must resist these pressures and make sure that patients benefit from the fruits of science, even in difficult times.

The use of placebo is especially beneficial in the midst of great anxiety, when the pressures to announce a cure or act on preliminary, but inconclusive data, are intense. A placebo relieves the temptation from all involved in the trial to engage in speculation and the worry that they are withholding what early (but often false) impressions may suggest is a promising drug. While remdesivir has shown promising activity in preclinical models of severe CoV infection, it is currently unknown whether it will prove safe and effective in treating severe COVID-19, both because of its uncertain antiviral efficacy and the unclear importance of ongoing viral replication versus an inflammatory process in disease pathogenesis in severe patients. Of note, remdesivir yielded positive results in preclinical models of Ebola virus infection but was inferior to two monoclonal antibody therapies in treated patients. ${ }^{15}$

During epidemics, as well as calls to treat everyone, other voices demand that vulnerable patients are not treated as 'guinea pigs'. Clinical trials use well thought through, tested and transparent scientific methods, and are undertaken as a joint endeavour between patients and health professionals within a clear ethical framework. Health emergencies raise additional ethical concerns but these have been carefully 
considered by various groups and clear guidance exists on the conduct of clinical research during health emergencies. ${ }^{20-22}$

The choice of the primary endpoint for a clinical trial is always challenging but has added difficulties when dealing with a disease with uncertain natural history, an over-stretched workforce, and the desire for an early answer. This requires an endpoint that is clinically meaningful, is based on simple data collection, has sufficient power, and will occur early. It also requires statistical methods that are robust to misspecification of design parameters, such as the endpoint frequency. We believe that our primary endpoint is both pragmatic and informative but we have included a range of secondary endpoints to show consistency with the primary endpoint, as recommended by the US Food and Drugs Administration for trials in influenza. ${ }^{23}$

\section{Trial Status}

Protocol version number $3.0,9^{\text {th }}$ February 2020

First patient, first visit $6^{\text {th }}$ February 2020; recruitment end date anticipated April 3, 2020

\section{Declarations}

\section{Acknowledgements}

Gilead Science provided previous preclinical and clinical data, and provided and shipped remdesivir and placebo free of charge.

Suggestions from WHO Novel Coronavirus R\&D.

Teddy Clinical Research Laboratory (Shanghai) Co., Ltd will act as central laboratory.

RT-PCR instrument and assay from Roche.

SMO assistance provided by Shanghai MedKey Med-Tech Development Co.,Ltd; Clinplus Co., Ltd; HANG ZHOU SIMO Co., Ltd; MEDPISON Co., Ltd.

Daniel Pan, Ni Li, Yinyan Xu, Zinan Yin, Jianwei Cui, Yindong Zhang, Zhiyan Xu, and Wang assisted with translation from Mandarin to English.

\section{Authors' contributions [31b]}

BC, YW, GHF, FZ, FH, PWH designed the trial.

TJ and GHF developed the statistical analysis plan

All authors read and approved the final manuscript. 


\section{Funding [4]}

- Emergency project for novel coronavirus pneumonia of the Ministry of Science and Technology (2020YFC0841300)

- Chinese Academy of Medical Sciences (CAMS) Innovation Fund for Medical Sciences (CIFMS 2018I2M-1-003)

- National Science Grant for Distinguished Young Scholars (81425001/H0104)

- The National Key Research and Development Program of China (2018YFC1200102),

- The Beijing Science and Technology Project (Z19110700660000)

- National Mega-projects for Infectious Diseases in China (2017ZX10103004 and 2018ZX10305409).

- TJ is funded by a NIHR Senior Research Fellowship (2015-08-001)

- PH is funded by the Bill and Melinda Gates Foundation, The Wellcome Trust, and the United Kingdom Department of Health and Social Care.

The study funders had no role in study design; collection, management, analysis, and interpretation of data; writing of the report; and the decision to submit the report for publication.

\section{Availability of data and materials [29]}

The datasets generated during and/or analysed during the current study will be made available.

\section{Ethics approval and consent to participate [24]}

This trial has been approved by the ethical review board of the China-Japan Friendship Hospital, No 2, East Yinghua Road, Chaoyang District, Beijing, 100029, China.

Informed consent will be obtained from all study participants.

\section{Consent for publication [32]}

Not applicable

\section{Competing interests [28]}

The authors declare that they have no competing interests.

\section{References}

1. Zhu N, Zhang D, Wang W, et al. A Novel Coronavirus from Patients with Pneumonia in China, 2019. N Engl J Med 2020.

2. Huang C, Wang Y, Li X, et al. Clinical features of patients infected with 2019 novel coronavirus in Wuhan, China. Lancet 2020. 
3. Chen N, Zhou M, Dong X, et al. Epidemiological and clinical characteristics of 99 cases of 2019 novel coronavirus pneumonia in Wuhan, China: a descriptive study. Lancet 2020.

4. Wang D, Hu B, Hu C, et al. Clinical Characteristics of 138 Hospitalized Patients With 2019 Novel Coronavirus-Infected Pneumonia in Wuhan, China. JAMA 2020.

5. Lo MK, Jordan R, Arvey A, et al. GS-5734 and its parent nucleoside analog inhibit Filo-, Pneumo-, and Paramyxoviruses. Sci Rep 2017; 7: 43395.

6. WHO. WHO R\&D Blueprint: informal consultation on prioritization of candidate therapeutic agents for use in novel coronavirus 2019 infection, Geneva, Switzerland, 24 January 2020., 2020.

7. Warren TK, Jordan R, Lo MK, et al. Therapeutic efficacy of the small molecule GS-5734 against Ebola virus in rhesus monkeys. Nature 2016; 531(7594): 381-5.

8. Jordan PC, Liu C, Raynaud P, et al. Initiation, extension, and termination of RNA synthesis by a paramyxovirus polymerase. PLoS Pathog 2018; 14(2): e1006889.

9. Tchesnokov EP, Feng JY, Porter DP, Gotte M. Mechanism of Inhibition of Ebola Virus RNA-Dependent RNA Polymerase by Remdesivir. Viruses 2019; 11(4).

10. Sheahan TP, Sims AC, Graham RL, et al. Broad-spectrum antiviral GS-5734 inhibits both epidemic and zoonotic coronaviruses. Sci Trans/ Med 2017; 9(396).

11. Wang M, Cao R, Zhang $L$, et al. Remdesivir and chloroquine effectively inhibit the recently emerged novel coronavirus (2019-nCoV) in vitro. Cell Res 2020.

12. Brown AJ, Won JJ, Graham RL, et al. Broad spectrum antiviral remdesivir inhibits human endemic and zoonotic deltacoronaviruses with a highly divergent RNA dependent RNA polymerase. Antiviral Res 2019; 169: 104541.

13. de Wit E, Feldmann F, Cronina J, et al. Prophylactic and therapeutic remdesivir (GS-5734) treatment in the rhesus macaque model of MERS-CoV infection. PNAS 2020.

14. Sheahan TP, Sims AC, Leist SR, et al. Comparative therapeutic efficacy of remdesivir and combination lopinavir, ritonavir, and interferon beta against MERS-CoV. Nat Commun 2020; 11(1): 222.

15. Mulangu S, Dodd LE, Davey RT, Jr., et al. A Randomized, Controlled Trial of Ebola Virus Disease Therapeutics. N Engl J Med 2019; 381(24): 2293-303.

16. Whitehead J, Stratton I. Group sequential clinical trials with triangular continuation regions. Biometrics 1983; 39(1): 227-36.

17. Schulz KF, Altman DG, Moher D. CONSORT 2010 statement: Updated guidelines for reporting parallel group randomised trials. J Pharmacol Pharmacother 2010; 1(2): 100-7.

18. Dimairo M, Coates E, Pallmann P, et al. Development process of a consensus-driven CONSORT extension for randomised trials using an adaptive design. BMC Med 2018; 16(1): 210.

19. Whitehead J. The design and analysis of sequential clinical trials.; 1997.

20. WHO. Ethics in epidemics, emergencies and disasters: Research, surveillance and patient care; 2015. 
21. Saxena A, Horby P, Amuasi J, et al. Ethics preparedness: facilitating ethics review during outbreaks recommendations from an expert panel. BMC Med Ethics 2019; 20(1): 29.

22. Nuffield Council on Bioethics. Research in global health emergencies: ethical issues, 2020.

23. U.S. Department of Health and Human Services Food and Drug Administration. Guidance for Industry. Influenza: Developing Drugs for Treatment and/or Prophylaxis, 2011. 